\title{
Clinically relevant doses of vitamin A decrease cortical bone mass in mice
}

\author{
Vikte Lionikaite1, Karin L Gustafsson', Anna Westerlund', Sara H Windahl', Antti Koskela², Juha Tuukkanen², \\ Helena Johansson³, Claes Ohlsson', H Herschel Conaway4, Petra Henning1,* and Ulf H Lerner1,* \\ ${ }^{1}$ Centre for Bone and Arthritis Research, Department of Internal Medicine and Clinical Nutrition, Institute for Medicine, Sahlgrenska Academy at \\ University of Gothenburg, Gothenburg, Sweden \\ 2Department of Anatomy and Cell Biology, Medical Research Center, University of Oulu, Oulu, Finland \\ ${ }_{3}^{3}$ nstitute for Health and Aging, Catholic University of Australia, Melbourne, Australia \\ ${ }^{4}$ Department of Physiology and Biophysics, University of Arkansas for Medical Sciences, Little Rock, Arkansas, USA \\ Correspondence should be addressed to P Henning or U H Lerner: petra.henning@gu.se or ulf.lerner@gu.se \\ *(P Henning and U H Lerner contributed equally to this work)
}

\section{Abstract}

Excess vitamin A has been associated with decreased cortical bone thickness and increased fracture risk. While most studies in rodents have employed high dosages of vitamin A for short periods of time, we investigated the bone phenotype in mice after longer exposure to more clinically relevant doses. For 1,4 and 10 weeks, mice were fed a control diet $(4.5 \mu \mathrm{g}$ retinyl acetate/g chow), a diet modeled from the human upper tolerable limit (UTL; $20 \mu \mathrm{g}$ retinyl acetate/g chow) and a diet three times UTL

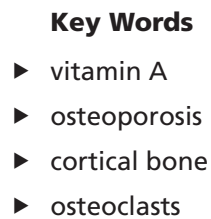
(supplemented; $60 \mu \mathrm{g}$ retinyl acetate/g chow). Time-dependent decreases in periosteal circumference and bone mineral content were noted with the supplemented dose. These reductions in cortical bone resulted in a significant time-dependent decrease of predicted strength and a non-significant trend toward reduced bone strength as analyzed by three-point bending. Trabecular bone in tibiae and vertebrae remained unaffected when vitamin A was increased in the diet. Dynamic histomorphometry demonstrated that bone formation was substantially decreased after 1 week of treatment at the periosteal site with the supplemental dose. Increasing amount of vitamin A decreased endocortical circumference, resulting in decreased marrow area, a response associated with enhanced endocortical bone formation. In the presence of bisphosphonate, vitamin A had no effect on cortical bone, suggesting that osteoclasts are important, even if effects on bone resorption were not detected by osteoclast counting, genes in cortical bone or analysis of serum TRAP5b and CTX. In conclusion, our results indicate that even clinically relevant doses of vitamin $A$ have a negative impact on the amount of cortical bone.

Journal of Endocrinology (2018) 239, 389-402

\section{Introduction}

Vitamin A is an essential nutrient consumed in the diet in the form of retinyl esters or beta carotene. Retinyl esters are transported by chylomicrons to the liver where they are converted to retinol and bound to retinol-binding protein and released into the blood stream. In target cells, retinol is converted to all-trans retinoic acid (ATRA), which is the hormonally active form of vitamin A. ATRA binds to cellular retinoic acid-binding protein and

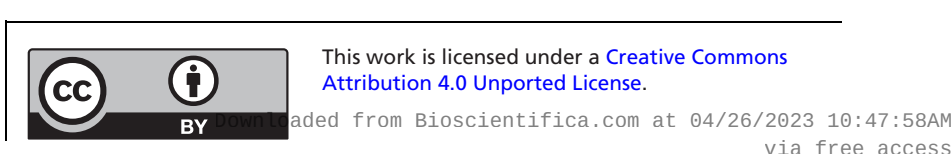


translocates to the nucleus where it ligates primarily to retinoic acid receptors, regulating gene transcription (reviewed in Tata 2002, Conaway et al. 2013, Henning et al. 2015, Green et al. 2016). ATRA can also diffuse to adjacent cells and have paracrine effects and acts as an important morphogen during embryonic development (Kam et al. 2012). Vitamin A plays a crucial role in various physiological functions, including immune system regulation, vision and cell growth and differentiation (reviewed in Wiseman et al. 2017).

In humans, increased vitamin A consumption and elevated serum retinol levels have been associated with increased bone fragility and fracture risk (Melhus et al. 1998, Whiting \& Lemke 1999, Feskanich et al. 2002, Michaelsson et al. 2003), suggesting that increased intake of vitamin A may be a risk factor for secondary osteoporosis. Although not all studies have consistently demonstrated the negative relationship between vitamin A and bone mass (for review see Conaway et al. 2013), a metaanalysis has shown that increased intake of vitamin A and elevated blood levels of retinol are associated with an increased risk of hip fracture (Wu et al. 2014). Interestingly, a dose-response analysis revealed that decreased levels of retinol also increased the risk of hip fracture (Wu et al. 2014).

Rodent studies have also shown detrimental effects of vitamin $\mathrm{A}$ on the skeleton. Rapid induction of hypervitaminosis A, either by feeding or by injecting retinoids, induces cortical bone loss in long bones of rats (Trechsel et al. 1987, Hough et al. 1988, Johansson et al. 2002, Kneissel et al. 2005, Lind et al. 2011, 2013, Wray et al. 2011) and mice (Kneissel et al. 2005), which is associated with decreased bone strength (Johansson et al. 2002, Lind et al. 2011). This reduction in the amount of cortical bone has been attributed to an increase in the number of periosteal osteoclasts (Trechsel et al. 1987, Hough et al. 1988, Kneissel et al. 2005, Lind et al. 2011). In trabecular bone, there have been fewer studies investigating the effects of vitamin A and the results have been inconsistent. In two studies, trabecular bone mineral density in the femur and the tibia was decreased by increased vitamin A intake (Lind et al. 2011, Wray et al. 2011), whereas in three other studies no effects on the trabecular bone mineral density of the humerus or tibia were observed (Johansson et al. 2002, Kneissel et al. 2005, Green et al. 2015). In the only study investigating the effect of increased dietary retinol on trabecular bone mass in vertebrae, it was found that vitamin A decreased bone mass, an effect associated with both an increased number of osteoclasts and a decreased number of osteoblasts (Yorgan et al. 2016).
Although numerous investigations have shown either stimulatory or inhibitory effects of vitamin A on osteoblast differentiation and function in vitro (reviewed in Conaway et al. 2013, Green et al. 2016), very few studies have analyzed effects of vitamin A on bone formation in vivo. Kneissel et al. and Lind et al. found that hypervitaminosis A decreased mineralizing surfaces on the periosteal side of cortical bone in the femur and tibia (Kneissel et al. 2005, Lind et al. 2013); however, mineralizing apposition rate was inhibited in one of these studies (Lind et al. 2013), but unaffected in the other (Kneissel et al. 2005).

The current recommended daily allowance (RDA) of vitamin A in adults is 900 and $700 \mu$ r retinol activity equivalents per day in males and females, respectively, while the UTL is $3000 \mu \mathrm{g} /$ day (Trumbo et al. 2001). With over one-third of the population in the USA taking dietary supplements (Bailey et al. 2011), excess vitamin A consumption/hypervitaminosis $\mathrm{A}$ is a potential health risk.

Although rodent studies have provided insight into changes in cortical bone caused by hypervitaminosis A, the doses (13 to at least 142 times higher than the human RDA diet) and the short durations (7-14 days) of the experiments are questionable reflections of human consumption. For this reason, the present study was designed to investigate the effects of lower, more clinically relevant dosages of vitamin A (4.5-13 times higher modeled from the human RDA) on bone phenotype in mice after longer exposure (1-10 weeks). We assessed in detail not only the effects of vitamin A on the amount of cortical bone, but also vitamin A's effects on trabecular bone in long bones as well as in vertebra, paying special attention to osteoclastic bone resorption and osteoblastic bone formation.

\section{Materials and methods}

\section{Animals and study design}

All animal experimental procedures were approved by the Ethics Committee at the University of Gothenburg and carried out in accordance to relevant guidelines. C57BL/6 female mice (Harlan Laboratories, Inc.; Taconic Bioscience) were received at 7-8 weeks of age and acclimatized for 1 week. C57BL6/J mice of 9-19 weeks, as used in this experiment, are sexually mature at the start of the experiments and growing less rapidly than mice aged 5-8 weeks. Mice were housed in groups of five at $22^{\circ} \mathrm{C}$ with a $12: 12 \mathrm{~h}$ light-darkness cycle and fed diets (Harlan Laboratories Inc.) containing either $4.5 \mu \mathrm{g}$ retinyl acetate/g chow (control (Harlan 2016) repelleted),

This work is licensed under a Creative Commons Attribution 4.0 Unported License. 
$20 \mu \mathrm{g}$ retinyl acetate/g chow (upper tolerable level; UTL) or $60 \mu \mathrm{g}$ retinyl acetate/g chow (supplemented) ad libitum for 4 ( $n=15 /$ group) or 10 weeks ( $n=10 /$ group). By using a 4.5- and 13-fold increased concentrations of retinyl acetate in the chow, we aimed to mimic the fold increase for upper tolerable and supplemented levels in humans calculated from the RDA. An additional 8-day experiment with three groups was performed, feeding 8- to 9-weekold mice control, supplemented or hypervitaminosis A diet (Hypervit A; 450 $\mu$ g retinyl acetate/g chow, $n=10$ /group).

A separate bisphosphonate experiment was performed on 8- to 9-week-old mice pre-treated with intraperitoneal (i.p.) injections of $200 \mu \mathrm{L}$ zoledronic acid (ZA; $200 \mu \mathrm{g} / \mathrm{kg}$; $0.02 \mu \mathrm{g} / \mu \mathrm{L}$; Frasenius Kabi AB, Uppsala, Sweden) or vehicle (saline) twice/week for 2 weeks ( $n=10 /$ group) (de Molon et al. 2015). They were then fed control ( $4.5 \mu \mathrm{g}$ retinyl acetate/g chow) or supplemented ( $60 \mu$ g retinyl acetate/g chow) vitamin A diets and the twice/week injections of ZA continued for 4 weeks.

Body weights were monitored throughout all experiments (Supplementary Fig. 1, see section on supplementary data given at the end of this article). At the termination of all experiments, mice were anesthetized with Ketador/Dexdomitor cocktail. Blood was collected from the subclavian artery. Serum was aliquoted and stored at $-80^{\circ} \mathrm{C}$ till further use. Mice were terminated via cervical dislocation. Liver was dissected and weighed. Vertebra (L3-L6), femurs and tibias were dissected, fixed in formalin for 3 days and stored in ethanol for later analysis.

The predesigned primary endpoints were to record the effect of vitamin A diets on cortical and trabecular bone mass. All assessments of the outcomes were done in total blinding of the investigators. Power analysis suggested that we needed at least eight mice per group to detect biologically significant effects.

\section{Serum analyses}

Serum retinol and retinyl ester (RE) analysis was carried out by Vital Analytical Services (Oslo, Norway) using high-performance liquid chromatography. Serum tartrate-resistant acid phosphatase (TRAP), C-terminal type I collagen (CTX) and osteocalcin (OCN) were measured using the MouseTRAP (TRAcP 5b) ELISA kit (Immunodiagnostic System), RatLaps (CTX-I) EIA ELISA kit (Immunodiagnostic System) and Osteocalcin ELISA kit (Immutopics, Inc.), respectively, following manufacturer's instructions.

$$
\text { http://joe.endocrinology-journals.org }
$$

\section{Quantitative PCR}

The vertebral body of the L3 and L6 (trabecular bone) and theflushed mid-diaphyseal tibias (corticalbone) werestored at $-80^{\circ} \mathrm{C}$ in RNAlater post killing. RNA was extracted using TRIzol reagent (Life Technologies) followed by the RNeasy mini kit (Qiagen). Single-strand cDNA was synthesized using a High-Capacity cDNA Reverse Transcription kit (Applied Biosystems). Quantitative real-time PCR (qPCR) analyses were performed by using predesigned Taqman Assays and Taqman Fast Advance Master Mix (Applied Biosystems). The following predesigned real-time PCR assays were used for gene expression analysis: Acp5 (Trap; Mm00475698_m1), Ctsk (Mm00484036_m1), Tnfsf11 (Rankl; Mm00441908_m1), Alpl (Alp; Mm00475834_m1), Tnfrs11a (Rank; Mm00437135_m1), Tnfrsf11b (Opg; Mm0043545_m1), Bglap (Osteocalcin; Mm01741771_g1),

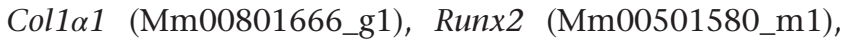
Sp7 (Osterix; Mm04209856_m1) (Applied Biosystems). The house-keeping gene $18 \mathrm{~S}$ was used as the endogenous control in all analyses. Data are displayed as fold change relative to control.

\section{Assessment of bone parameters}

\section{Peripheral quantitative computed tomography (pQCT) and microcomputed tomography ( $\mu C T)$}

Peripheral quantitative computed tomography (pQCT) was run on the femur and tibia with the pQCT XCT RESEARCH M (version 4.5B; Norland). The voxel size was $70 \mu \mathrm{m}$ isotropically. The growth plate was located by a scout scan, and the boundary of the metaphysis and growth plate was used as a reference point for trabecular and cortical scans. Trabecular bone mineral density (Tb.BMD) was determined with a single metaphyseal scan of the proximal tibia, at a distance $2.6 \%$ of the total tibia bone length distal from the growth plate, or of the distal femur, corresponding to a distance $3 \%$ of the total femur bone length proximal from the growth plate. Trabecular bone region was defined as the inner $45 \%$ of the total crosssectional area to avoid contamination with cortical bone. Cortical bone parameters were analyzed by a single scan in the approximate mid-diaphyseal region of the tibia, at a distance $30 \%$ of the total tibia bone length distal from the proximal growth plate or of the femur, at a distance $36 \%$ of the total femur bone length proximal from the distal growth plate. Cortical bone mineral content is presented as $\mathrm{mg} / \mathrm{mm}$ bone length calculated from the diaphyseal scan. Cortical thickness was determined by the software (Stratec XCT, Research M pQCT v. 6.20C) as the average 
thickness using a circular model with the threshold set to $710 \mathrm{mg} / \mathrm{cm}^{3}$. Bone length was measured using a caliper.

Microcomputed tomography $(\mu \mathrm{CT})$ analysis was performed on the vertebra (L5) using the Skyscan 1072 scanner (Bruker MicroCT, Aartselaar, Belgium) and imaged with an X-ray tube voltage of $50 \mathrm{kV}$ and current of $201 \mu \mathrm{A}$ with an $0.5 \mathrm{~mm}$ aluminum filter. Transverse scanning angular rotation was $180^{\circ}$ and the angular increment of $0.70^{\circ}$. The voxel size was $13.5 \mu \mathrm{m}$ isotropically. The NRecon software (Burker) was used for image reconstruction following established guidelines (Bouxsein et al. 2010).

\section{Histomorphometry and measurement of mechanical strength}

For the measure of dynamic bone parameters, mice were injected (i.p.) with $100 \mu \mathrm{L}$ of calcein $(50 \mathrm{mg} / \mathrm{kg}) 8$ and 1 day before termination. The whole femurs were fixed in $4 \%$ paraformaldehyde, followed by dehydration in $70 \%$ ethanol and embedded in methyl meta-acrylate. Twenty-five micrometer transverse sections of cortical bone from the approximate mid-diaphyseal region were cut and periosteal and endocortical mineralized surface /bone surface (MS/BS), mineral apposition rate (MAR) and bone formation rate/bone surface (BFR/BS) were analyzed.

For static cellular bone parameters, femurs were fixed in $4 \%$ phosphate-buffered paraformaldehyde, decalcified in 10\% EDTA in Tris-buffer $\mathrm{pH} 6.95$ and embedded in paraffin. Coronal sections were stained with heamatotoxylin and TRAP-positive osteoclasts were detected by the Naphol AS-BI method. Trabecular bone osteoclasts were assessed at the distal and proximal femur in the metaphyseal area, not including osteoclasts near the growth plate. Cortical bone osteoclasts were counted in the diaphysis, excluding the metaphysis.

Histological measurements of marrow area (Ma.Ar), total area (Tt.Ar) and cortical bone area (Ct.Ar) were acquired from sections used for dynamic histomorphometry.

All parameters (dynamic and static) were measured using the Osteomeasure Histomorphometry System (OsteoMetrics) following the guidelines of the American Society for Bone and Mineral Research (Dempster et al. 2013).

Mechanical strength was assessed in the tibias from 10-week control and supplemented vitamin A mice using the three-point bending test. Supports were placed $\pm 2.75-2.8 \mathrm{~mm}$ from $50 \%$ of the total length of the bone, totaling the span length of $5.5 \mathrm{~mm}$. Tests were performed at a loading speed of $0.155 \mathrm{~mm} / \mathrm{s}$ with a mechanical testing machine (Instron 3366, Instron) at mid-tibia, with the press head loaded on the medial side. Results were calculated with custom-made Excel macros based on the computer recorded load deformation raw data curves before the first point of permanent deformation, produced by Bluehill 2 software v2.6 (Insitron). From the raw data curves, the $F(\max )$ is the maximum loading force (N) applied till breaking, and stiffness $(\mathrm{N} / \mathrm{mm})$ was the slope of the load deformation curve.

\section{Statistical analyses}

Figures and tables are presented as mean \pm standard error of the mean (s.e.M.). D'Agostino-Pearson omnibus normality test was used to assess normality of data and equal variance was assumed. Student's t-test and one-way ANOVA with Dunnett's post hoc test were used to analyze treatment effect. Two-way ANOVA for interaction was used to study the time-dependent effect of vitamin A treatment and the result of ZA treatment. No adjustment was made for multiple comparison testing other than Dunnett's. 95\% CIs of the effect are displayed where appropriate. GraphPad Prism 7 statistical software v7.02 (GraphPad Software, Inc) was used with $P<0.05$ considered statistically significant. A multivariable linear regression model was fitted to 4- and 10-week treatment data using both dosage (4.5, 20 and $60 \mu \mathrm{g}$ retinyl acetate/g chow) and duration (4 and 10 weeks) of treatment as independent variables, and bone mineral content (BMC), cortical thickness and periosteal and endocortical circumference as dependent variable in each model. The interaction between dosage and duration was used as an additional independent variable. In a linear regression model, the association between dosage $(4.5,20$ and $60 \mu \mathrm{g} / \mathrm{g})$ and cortical bone mass is linear and the model cannot detect a non-linear association, i.e. the reported decrease of cortical bone mass per $10 \mu \mathrm{g} / \mathrm{g}$ increase is estimated from the overall association between cortical bone mass and the dosage in the three treatment groups.

\section{Results}

Vitamin A-treated mice appear healthy with increased relative liver weights and increased serum retinol and retinyl ester levels

After 4 and 10 weeks of treatment with UTL or supplemented doses of vitamin A, mice appeared healthy with no significant difference in body weight change between treatment groups and control (Table 1 and Supplementary Fig. 1). Liver weight, as percentage of body weight, was increased at 4 and 10 weeks in the supplemented dose group compared to control mice

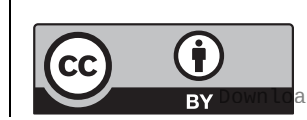

This work is licensed under a Creative Commons Attribution 4.0 Unported License. 
A
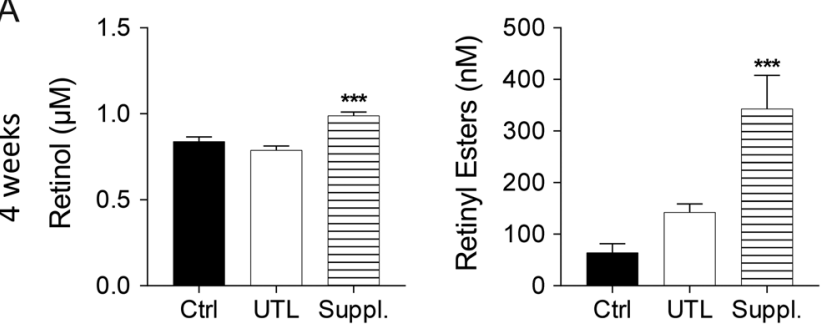

B
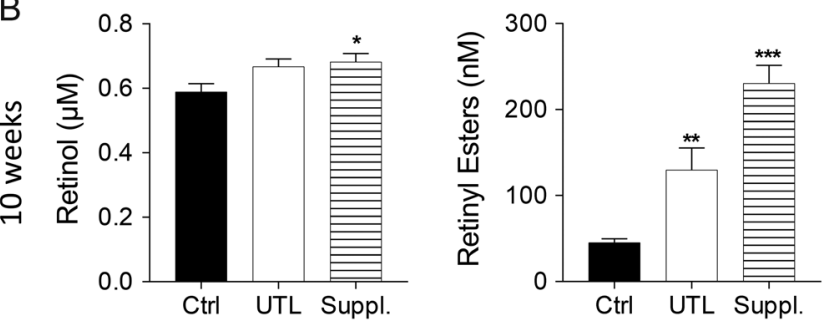

Figure 1

Vitamin A supplementation increases serum retinol and retinyl ester (RE) levels. Serum retinol and RE at 4 (A) and 10 (B) weeks. Values given as mean \pm S.E.M. ( $n=10 /$ group). 1-way ANOVA followed by Dunnett's multiple comparison test vs control, ${ }^{*} P<0.05, * * P<0.01, * * * P<0.001$.

(Table 1). Bone length of the tibia and femur was not significantly affected by the UTL or supplemental doses compared to controls (Table 1).

Mice fed control chow containing $4.5 \mu \mathrm{g}$ retinyl acetate/g chow for 4 weeks had serum levels of $0.84 \pm 0.03 \mu \mathrm{M}$ retinol and $65 \pm 17 \mathrm{nM}$ retinyl esters, which is similar to what has been previously observed in $\mathrm{C} 57 \mathrm{BL} / 6$ mice (Obrochta et al. 2014). Serum retinol was increased significantly by the supplemented dose of vitamin A in comparison to control at both time points, with the UTL diet showing no significant change (Fig. 1). The serum $\mathrm{RE}$ (the sum of retinyl linoleate, retinyl palmitate, retinyl oleate, retinyl stearate) levels were significantly higher than the RE in mice fed control chow after both 4 and 10 weeks of supplemental vitamin A diet. Mice fed UTL chow displayed significantly increased serum RE levels at 10 weeks (Fig. 1).

\section{Vitamin A decreases cortical bone parameters in a time-dependent manner}

After 4 weeks of supplemental vitamin A diet, cortical BMC, cortical thickness and cortical bone mineral density (BMD) were lower in the tibia (Fig. 2A, B and C) as assessed by pQCT. After 10 weeks of treatment, the effects on cortical BMC were significantly more pronounced (Fig. 2A). Both periosteal and endocortical circumferences were significantly decreased after 4 weeks with the supplemented diet (Fig. 2D and E). The decrease in periosteal circumference progressively increased after 10 weeks (Fig. 2D). Similar results were also obtained in the femur (Supplementary Fig. 2A, B, C, D, E and F). The UTL diet caused a significant decrease in periosteal circumference in both the tibia (Fig. 2D) and the femur (Supplementary Fig. 2F) over time.

These changes in cortical bone led to a significant decrease in mean polar moment of inertia (predicted strength) assessed by pQCT, in both the tibia (Fig. 2F) and the femur (Supplementary Fig. 2F) after 4 and 10 weeks of treatment with the supplemented diet, but not the UTL diet. Formal strength testing (three-point bending)

Table 1 Final body weight, relative liver weight, femur and tibia length.

\begin{tabular}{l}
\hline Final body weight (g) \\
Control \\
UTL \\
Supplemented \\
Liver weight $(\%)$ \\
Control \\
UTL \\
Supplemented \\
Tibia length (mm) \\
Control \\
UTL \\
Supplemented \\
Femur length (mm) \\
Control \\
UTL \\
Supplemented
\end{tabular}

4 weeks $(n=15 /$ group $)$
$18.2 \pm 0.3$
$18.6 \pm 0.3$
$18.2 \pm 0.3$
$4.3 \pm 0.09$
$4.6 \pm 0.07$
$4.8 \pm 0.13 * *$
$16.8 \pm 0.1$
$16.9 \pm 0.08$
$16.9 \pm 0.13$
$14.4 \pm 0.11$
$14.6 \pm 0.09$
$14.5 \pm 0.1$

\begin{tabular}{c}
\hline 10 weeks $(n=10 /$ group $)$ \\
$20.9 \pm 0.5$ \\
$20.2 \pm 0.5$ \\
$20.7 \pm 0.5$ \\
$4.2 \pm 0.06$ \\
$4.5 \pm 0.07$ \\
$4.6 \pm 0.12 * *$ \\
$17.3 \pm 0.15$ \\
$17.3 \pm 0.19$ \\
$17.6 \pm 0.17$ \\
$14.8 \pm 0.17$ \\
$14.9 \pm 0.15$ \\
$15.0 \pm 0.12$
\end{tabular}

Final body weight, liver weight ( $\%$ of body weight) and tibia and femur length after 4 and 10 weeks of UTL and supplemented diets. Values given as mean \pm S.E.M.

$* * P<0.01$ : 1-way ANOVA followed by Dunnett's multiple comparison test vs respective control.

http://joe.endocrinology-journals.org https://doi.org/10.1530/JOE-18-0316
(C) 2018 The authors Published by Bioscientifica Ltd. Printed in Great Britain

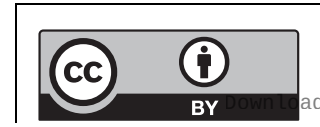

This work is licensed under a Creative Commons Attribution 4.0 Unported License. 

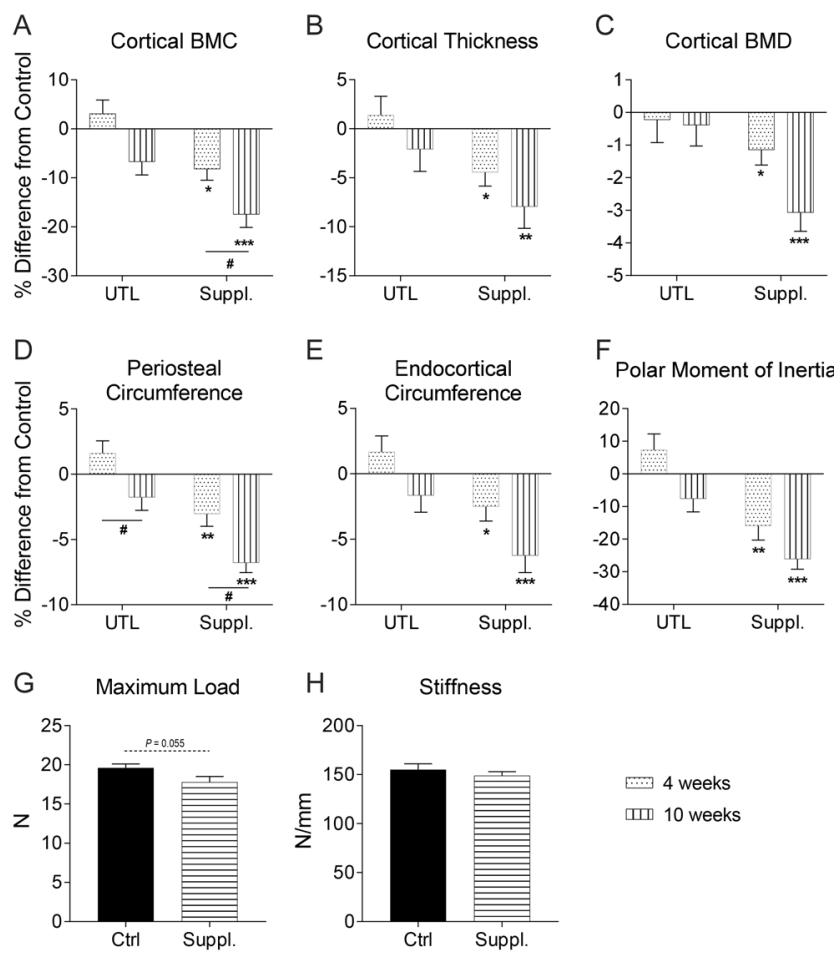

IIII 10 weeks

\section{Figure 2}

Supplemented vitamin A dose, but not UTL dose, affects cortical bone parameters of the tibia in a time-dependent manner and decreases predicted bone strength. UTL and supplemented diet effect on (A) cortical bone mineral content (BMC), (B) cortical thickness, (C) cortical bone mineral density (BMD), (D) periosteal circumference, $(E)$ endocortical circumference, and (F) polar moment of inertia of the tibia. pQCT results in \% difference vs respective control. Three-point bending of the tibia after 10 weeks of supplemented diet (G) maximum load applied till failure $(P=0.055)$ and $(H)$ stiffness. $(A, B, C, D, E$ and $F)$ Student's $t$-test vs respective controls, ${ }^{*} P<0.05, * * P<0.01, * * * P<0.001$. 2-way ANOVA for interaction, $\# P<0.05, \# P<0.01 . n=15$ /group at 4 weeks, $n=10 /$ group at 10 weeks. ( $G$ and H) Student's $t$-test, $n=10 /$ group. All values displayed as mean \pm S.E.M.

was performed on tibias from 10-week supplemental diet, since the phenotype observed was more pronounced than at 4 weeks. Supplemented vitamin A diet resulted in a trend of lower bone strength $(-9 \% ; P=0.055$; Fig. $2 \mathrm{G})$, while the stiffness remained unchanged between the two groups (Fig. 2H).
Linear regression analyses, using data from the three treatment groups $(4.5,20$ and $60 \mu \mathrm{g}$ retinyl acetate/g chow) and both treatment time points ( 4 and 10 weeks), showed that femur cortical BMC and thickness, as well as periosteal and endocortical circumference are significantly associated with the dosage of vitamin A when the data are adjusted for treatment time (Table 2). There was no statistical significant interaction between dosage and time for any cortical bone parameter $(P>0.14)$.

\section{Supplemental vitamin A diet does not affect trabecular bone}

Trabecular bone mass in tibia was not significantly changed after 4 and 10 weeks of UTL and supplemented vitamin A chow compared to control, as assessed by pQCT (Table 3). To further analyze trabecular bone, microCT analysis was performed on the L5 vertebra. Results demonstrated that trabecular bone volume fraction in the vertebrae was not significantly affected with the supplemented vitamin A diet compared to control in the present study (Table 3).

\section{Expression analysis of osteoclastic and osteoblastic genes in cortical bone}

The mRNA expression of osteoclastic genes in cortical bone of the tibia (Acp5, Ctsk, Tnfrsf11a, Tnfsf11, Tnfrsf11b) was not affected after 4 or 10 weeks of supplemented diet (Table 4). At 4 weeks, the mRNA expression of the osteoblastic genes osteocalcin (Bglap) and alkaline phosphatase $(A l p l)$ were decreased after both supplemented (Table 4) and UTL (data not shown) dosages of vitamin A, whereas other osteoblastic genes, such as Col1a1, Runx2, and $S p 7$, were not significantly regulated.

In vertebra, the mRNA expression of Acp5, Ctsk, Tnfrsf11a, Tnfsf11, Tnfrsf11b, Bglap, Alpl, Col1a, Runx2 and $S p 7$ were not significantly regulated by the supplemented diet group after 10 weeks (data not shown).

Table 2 Linear regression analysis indicates that increases of vitamin A in the chow is significantly associated with lower cortical bone parameters when adjusted for number of weeks of treatment.

\begin{tabular}{lc}
\hline Cortical BMC $(\mathrm{mg} / \mathrm{mm})$ & Change per $\mathbf{1 0} \boldsymbol{\mu g} / \mathbf{g}$ increase in dose $(95 \% \mathrm{Cl})$ \\
Cortical thickness $(\mu \mathrm{m})$ & $-0.016(-0.023,-0.0085)^{* * *}$ \\
Periosteal circumference $(\mathrm{mm})$ & $-0.0012(-0.0023,-0.0001)^{*}$ \\
Endocortical circumference $(\mathrm{mm})$ & $-0.040(-0.053,-0.026)^{* * *}$ \\
\hline
\end{tabular}

Values given as change per $10 \mu \mathrm{g} / \mathrm{g}$ increase in dose with $95 \%$ confidence intervals $(95 \% \mathrm{Cl})$.

$* P<0.05, * * * P<0.001$.

http://joe.endocrinology-journals.org https://doi.org/10.1530/JOE-18-0316
(C) 2018 The authors Published by Bioscientifica Ltd. Printed in Great Britain

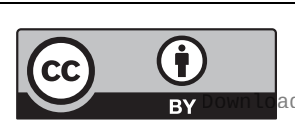

This work is licensed under a Creative Commons Attribution 4.0 Unported License. 
Serum resorption and formation markers indicate no effect on osteoclast or osteoblast activity

Analysis of serum TRAP5b and CTX indicated no significant differences in osteoclast activity at 4 or 10 weeks of supplemented vitamin A diet (Supplementary Table 1). Osteoblast activity also appeared to be unchanged when analyzed by serum levels of osteocalcin (Supplementary Table 1).

\section{Histomorphometric analysis indicates no significant change in the number of osteoclasts}

TRAP stained longitudinal sections of the femurs of mice exposed to supplemented diet for 10 weeks revealed no significant change in the number of osteoclasts present on either the periosteal, endocortical, or trabecular bone (Supplementary Table 2).

\section{Dynamic histomorphometry shows that long-term vitamin A transiently increases endocortical bone formation rate with no effect on the periosteal surface}

Dynamic histomorphometry measurements showed that endocortical MAR and bone formation rate (BFR/BS) were increased in the femur after 4 weeks of supplemented diet, but no significant effect on mineralization surfaces (MS/BS) was noted in the present study (Table 5). After 10 weeks of the diet, these increases were no longer observed; however, marrow area was decreased by $31 \%$ compared to controls (Table 5). In contrast, MS/BS, MAR or BFR/BS at the periosteal surface were not significantly affected at 4 and 10 weeks, although the total area was decreased by $19 \%$ after 10 weeks (Table 5).
Short duration supplemented and hypervitaminosis A diet decrease cortical bone parameters

We observed that the periosteal and endocortical circumferences, as well as cortical BMC, were reduced after 8 days in mice fed supplemented vitamin A diet when assessed by pQCT (Table 6).

Hypervitaminosis A treatment $(450 \mu \mathrm{g}$ retinyl acetate/g chow) induced a much larger effect on bone phenotype than those observed with supplemented diet, illustrated by more pronounced decreases in several cortical bone parameters in the femur, including cortical BMC and cortical thickness, as well as periosteal and endocortical circumferences, without effects on longitudinal bone growth (Table 6). We could not detect any significant effect on the trabecular BMD (95\% CI of effect: $-78.8,23.2)$ in the metaphyseal area of the femur, as assessed by pQCT, by hypervitaminosis A diet (Table 6). Similar cortical and trabecular parameters were also observed in the tibia (data not shown). Mice treated with the hypervitaminosis A diet had decreased final body weight and increased relative liver weight compared to control (Table 6).

\section{Short duration hypervitaminosis $\mathbf{A}$ increases the number of periosteal osteoclasts and decreases endocortical osteoclasts}

Static histomorphometry of femurs in mice fed a hypervitaminosis A diet showed increases in the number of osteoclasts on periosteal bone and decreases on endocortical bone, but no effect on trabecular osteoclast numbers (Fig. 3A, B, C, D and E). In contrast, no significant changes in osteoclast numbers were observed in mice fed the supplemented vitamin A diet (Fig. 3C, D and E).

Table 3 UTL and supplemental vitamin A diets did not affect trabecular bone.

\begin{tabular}{|c|c|c|c|c|c|}
\hline & \multirow{2}{*}{$\begin{array}{c}\text { Tibia } \\
\text { Tb.BMD }\end{array}$} & \multicolumn{4}{|c|}{ Vertebra } \\
\hline & & $\mathrm{BV} / \mathrm{TV}$ & Tb.N & Tb.Th & Tb.Sp \\
\hline 4 weeks & ( $n=15 /$ group) & \multicolumn{4}{|c|}{ ( $n=10 /$ group) } \\
\hline Control & $209 \pm 6$ & $22.8 \pm 1.1$ & $4.96 \pm 0.2$ & $46 \pm 1$ & $93 \pm 3$ \\
\hline UTL & $211 \pm 6(-20.7,24.6)$ & - & - & - & - \\
\hline Supplemented & $219 \pm 9(-12.1,33.2)$ & $23.3 \pm 1.2(-2.91,3.75)$ & $5.22 \pm 0.2(-0.33,0.85)$ & $45 \pm 1(-4.02,0.96)$ & $96 \pm 4(-7.94,13.4)$ \\
\hline 10 weeks & ( $n=10 /$ group) & \multicolumn{4}{|c|}{$(n=10 /$ group $)$} \\
\hline Control & $200 \pm 8$ & $22.1 \pm 1.0$ & $4.77 \pm 0.2$ & $47 \pm 0.7$ & $158 \pm 4$ \\
\hline UTL & $210 \pm 12(-26.3,45.0)$ & - & - & - & - \\
\hline Supplemented & $179 \pm 12(-56.5,14.8)$ & $22.4 \pm 1.4(-3.37,3.79)$ & $5.02 \pm 0.3(-0.53,1.04)$ & $45 \pm 1(-4.54,0.43)$ & $155 \pm 5(-16.2,10.2)$ \\
\hline
\end{tabular}

http://joe.endocrinology-journals.org https://doi.org/10.1530/JOE-18-0316
(C) 2018 The authors Published by Bioscientifica Ltd. Printed in Great Britain

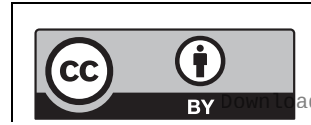

This work is licensed under a Creative Commons Attribution 4.0 Unported License. 
Table 4 Expression analysis of osteoclastic and osteoblastic genes in cortical bone after 4 and 10 weeks of supplemented vitamin A diet.

\begin{tabular}{|c|c|c|}
\hline & \multicolumn{2}{|c|}{4 week } \\
\hline & Control & Supplemented \\
\hline Acp5 (Trap) & $1.00 \pm 0.16$ & $0.78 \pm 0.07$ \\
\hline Ctsk & $1.00 \pm 0.17$ & $0.81 \pm 0.08$ \\
\hline Tnfrsf11a (Rank) & $1.00 \pm 0.12$ & $0.97 \pm 0.09$ \\
\hline Tnfsf11 (Rankl) & $1.00 \pm 0.17$ & $0.90 \pm 0.07$ \\
\hline Tnfrsf11b (Opg) & $1.00 \pm 0.20$ & $0.79 \pm 0.12$ \\
\hline Bglap (Ocn) & $1.00 \pm 0.18$ & $0.49 \pm 0.08^{*}$ \\
\hline$A / p l$ & $1.00 \pm 0.15$ & $0.63 \pm 0.06$ * \\
\hline Col1 $\alpha 1$ & $1.00 \pm 0.17$ & $0.82 \pm 0.07$ \\
\hline Runx2 & $1.00 \pm 0.20$ & $0.60 \pm 0.07$ \\
\hline Sp7 (Osterix) & $1.00 \pm 0.13$ & $1.35 \pm 0.20$ \\
\hline
\end{tabular}

\begin{tabular}{c}
\hline Cl of effect \\
\hline$-0.58,0.14$ \\
$-0.59,0.21$ \\
$-0.35,0.29$ \\
$-0.49,0.29$ \\
$-0.69,0.27$ \\
$-0.93,-0.09$ \\
$-0.70,-0.04$ \\
$-0.57,0.21$ \\
$-0.84,0.04$ \\
$-0.14,0.85$ \\
\hline
\end{tabular}

\begin{tabular}{ccc}
\hline \multicolumn{2}{c}{$\mathbf{1 0}$ week } \\
\hline Control & & Supplemented \\
\hline $1.00 \pm 0.10$ & & $0.89 \pm 0.08$ \\
$1.00 \pm 0.11$ & & $0.83 \pm 0.06$ \\
$1.00 \pm 0.10$ & & $0.90 \pm 0.05$ \\
$1.00 \pm 0.13$ & & $1.03 \pm 0.13$ \\
$1.00 \pm 0.06$ & & $1.39 \pm 0.19$ \\
$1.00 \pm 0.10$ & & $1.05 \pm 0.18$ \\
$1.00 \pm 0.07$ & & $1.13 \pm 0.17$ \\
$1.00 \pm 0.10$ & & $1.10 \pm 0.17$ \\
$1.00 \pm 0.07$ & & $1.06 \pm 0.06$ \\
$1.00 \pm 0.06$ & $1.18 \pm 0.11$ \\
\hline
\end{tabular}

$\mathrm{Cl}$ of effect

$-0.38,0.17$

$-0.44,0.09$

$-0.34,0.14$

$-0.35,0.42$

$-0.03,0.82$

$-0.37,0.48$

$-0.25,0.51$

$-0.31,0.52$

$-0.14,0.25$

Osteoclastic (Acp5, Ctsk, Tnfrsf11a, Tnfsf11, and Tnfrsf11b) and osteoblastic (Bglap, Alpl, Col1 11 , Runx2, and Sp7) genes at 4 and 10 weeks of supplemented vitamin A treatment and the $95 \% \mathrm{Cl}$ of the effect. Data is displayed as fold change relative to control, $n=10 / g$ roup, mean \pm s.E.M., Student's $t$-test vs control.

$* P<0.05$.

\section{Short duration hypervitaminosis A increases osteoclastic gene expression and serum Trap5b}

Cortical bone gene expression in tibia of the osteoclast genes Acp5, Ctsk and Tnfsf11 increased with hypervitaminosis A treatment compared to control, but was not significantly changed by supplemental vitamin A diet compared to control (Fig. 3F, G and H). This stimulatory effect by hypervitaminosis A was mimicked by serum TRAP5b analysis (Fig. 3N).

\section{Short-term supplemented dose of vitamin A affects periosteal and endocortical bone formation}

Osteoblast genes (Tnfrsf11b, Alpl, Bglap, Runx2 and Sp7) remained unchanged with both the supplemented dose and hypervitaminosis A treatment (Fig. 3I, J, K, L and M). Importantly, dynamic histomorphometry in mice fed the supplemented vitamin A diet for 8 days showed a pronounced decrease in periosteal BFR $(-61 \%, P<0.05$; Table 7). In contrast, endocortical BFR was increased by the supplemented diet (Table 7).

\section{Vitamin A effects on cortical bone were not observed in the presence of bisphosphonate} treatment

No significant effects of vitamin A on periosteal or endocortical circumferences was observed when ZA-treated mice were fed supplemented vitamin A chow (Fig. 4D and E). Treatment with ZA resulted in decreased bone length (Fig. 4A) and increased trabecular BMD and cortical thickness (Fig. 4B and C) compared to vehicle controls.

$$
\text { http://joe.endocrinology-journals.org }
$$

Mice treated with ZA appeared healthy throughout the experiment and the treatment did not affect body weight change (Supplementary Fig. 1C).

\section{Discussion}

The main findings of this study are as follows: (1) Experiments of 10-week duration with diets containing either 20 or $60 \mu$ g retinyl acetate/g of chow resulted in dose-dependent increases of circulating levels of RE equal to 130 and $231 \mathrm{nM}$, respectively. These are levels below or close to levels suggested for hypervitaminosis A in humans (200 nM) (Krasinski et al. 1989). (2) Increases in vitamin A levels time-dependently decreased cortical bone mass resulting in a trend toward decreased strength, (3) supplemental vitamin A diet did not significantly affect trabecular bone phenotype in the tibia or vertebra, (4) increased osteoclastic bone resorption, in combination with an early decrease in periosteal bone formation resulted in decreased periosteal circumference and (5) enhanced endocortical bone formation resulted in decreased marrow area and endocortical circumference.

The predominant form of vitamin $\mathrm{A}$ in the serum is retinol with normal physiological levels of $2-4 \mu \mathrm{M}$ in humans and $1 \mu \mathrm{M}$ in mice (Krasinski et al. 1989, Michaelsson et al. 2003, O'Byrne \& Blaner 2013, Obrochta et al. 2014). In the present study, mice fed the supplemental diet had increased serum retinol levels. This method of determining vitamin A status is frequently used in human assessment; however, retinol levels in the serum are not reflective of vitamin A status unless there is a deficiency or surplus of the nutrient. Serum retinyl

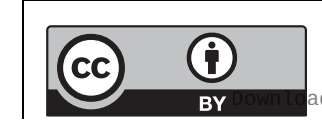


Table 5 Dynamic histology shows vitamin A transiently increases endocortical bone formation after 4 weeks of supplemented vitamin A diet. Histological analysis indicates smaller marrow area and total area after 10 weeks of supplemental diet.

\begin{tabular}{l} 
\\
\hline Periosteal \\
MS/BS $(\%)$ \\
MAR $(\mu \mathrm{m} /$ day $)$ \\
BFR/BS $\left(\mu \mathrm{m}^{3} / \mu \mathrm{m}^{2} /\right.$ day $)$ \\
Endocortical \\
MS/BS $(\%)$ \\
MAR $(\mu \mathrm{m} /$ day $)$ \\
BFR/BS $\left(\mu \mathrm{m}^{3} / \mu \mathrm{m}^{2} /\right.$ day $)$ \\
Ma.Ar $\left(\mathrm{mm}^{2}\right)$ \\
Tt.Ar $\left(\mathrm{mm}^{2}\right)$ \\
Ct.Ar $\left(\mathrm{mm}^{2}\right)$
\end{tabular}

\begin{tabular}{ccl}
\multicolumn{2}{c}{ 4 weeks } \\
\cline { 1 - 1 } Control & & Supplemented \\
$30.5 \pm 2.27$ & & $22.0 \pm 3.44$ \\
$0.72 \pm 0.06$ & & $0.59 \pm 0.07$ \\
$0.22 \pm 0.03$ & & $0.19 \pm 0.06$ \\
& & \\
$76.4 \pm 1.40$ & & $81.2 \pm 2.83$ \\
$2.18 \pm 0.20$ & & $2.82 \pm 0.10^{*}$ \\
$1.66 \pm 0.15$ & & $2.28 \pm 0.08 * *$ \\
$0.52 \pm 0.03$ & & $0.55 \pm 0.03$ \\
$0.96 \pm 0.04$ & & $0.96 \pm 0.04$ \\
$0.44 \pm 0.01$ & & $0.41 \pm 0.01$
\end{tabular}

\begin{tabular}{c}
\hline Control \\
\hline $31.2 \pm 3.85$ \\
$0.67 \pm 0.04$ \\
$0.23 \pm 0.04$ \\
$74.0 \pm 3.20$ \\
$1.38 \pm 0.05$ \\
$1.02 \pm 0.05$ \\
$0.74 \pm 0.03$ \\
$1.48 \pm 0.05$ \\
$0.74 \pm 0.03$ \\
\hline
\end{tabular}

10 wee

Supplemented

Interaction
n.s.
n.s.
n.s.
n.s.
$P<0.05$
$P<0.05$
$P<0.001$
$P<0.01$
n.s.

Femur periosteal and endocortical mineralized surface/bone surface (MS/BS), mineral apposition rate (MAR), and bone formation rate/bone surface (BFR/BS) obtained from dynamic histomorphometry, and marrow area (Ma.Ar), total area (Tt.Ar), and cortical bone area (Ct.Ar) after 4 and 10 weeks of supplemented vitamin A diet. Values represented as mean \pm S.E.M., $n=10 /$ group, Student's $t$-test vs control, 2-way ANOVA for interactions, n.s. indicates no statistical significance.

${ }^{*} P<0.05, * * P<0.01, * * * P<0.001$

esters have been shown to be a more precise measurement of vitamin A status (Croquet et al. 2000). The normal physiological levels of retinyl esters in humans are in the range of 50-200 nM (Krasinski et al. 1989, Ballew et al. 2001), and it has been suggested that RE levels over $200 \mathrm{nM}$ or exceeding $10 \%$ of total serum vitamin A (retinol and RE) may indicate excess vitamin A stores and potential vitamin A toxicity (Krasinski et al. 1989), although a specific cutoff has been difficult to determine (Ballew et al. 2001). In our experiments, 4 and 10 weeks of supplemented vitamin A diet resulted in RE levels of $343 \pm 65 \mathrm{nM}$ and $231 \pm 21 \mathrm{nM}$, respectively, which exceeds the suggested RE threshold set for humans and indicates potential vitamin A excess.

The UTL dose used in the present study ( $20 \mu \mathrm{g}$ retinyl acetate/g chow) resulted in RE levels of $130-143 \mathrm{nM}$ at 4 and 10 weeks. This dose, modeled after the human UTL, by definition should be the maximum vitamin A consumption that does not cause ill effects. Although no statistically significant bone phenotype compared to control was observed, there was a trend for effects on all cortical bone parameters observed after 10, but not after 4 weeks, which is reflected by a significant decrease of periosteal circumference in the femur and tibia at 10 weeks when compared to the circumference at 4 weeks. The effects at 10 weeks were also similar, though not as pronounced as those noted with the supplemented diet ( $60 \mu \mathrm{g}$ retinyl acetate/g chow) at 10 weeks.

Linear regression analysis showed that there was a significant association of increased vitamin A dosage $(4.5,20$ and $60 \mu \mathrm{g}$ retinyl acetate/g chow) and decreased cortical bone parameters. These observations indicate that even small increases of the vitamin A dose may negatively affect cortical bone parameters although the effects of the

Table 6 Short duration supplemented vitamin A diet decreased endocortical circumference and hypervitaminosis A diet decreased cortical bone parameters.

\begin{tabular}{|c|c|c|c|}
\hline & Control & Supplemented & Hypervitaminosis A \\
\hline Final body weight (g) & $18.6 \pm 0.3$ & $18.1 \pm 0.2$ & $17.3 \pm 0.2 * *$ \\
\hline Liver weight (\%) & $4.9 \pm 0.1$ & $4.9 \pm 0.09$ & $5.3 \pm 0.1 * *$ \\
\hline Femoral length (mm) & $14.2 \pm 0.07$ & $14.2 \pm 0.05$ & $14.3 \pm 0.07$ \\
\hline Tb. BMD $\left(\mathrm{mg} / \mathrm{cm}^{3}\right)$ & $387.8 \pm 9.83$ & $347.3 \pm 17.3$ & $360.0 \pm 15.5$ \\
\hline Endocortical circumference (mm) & $3.28 \pm 0.03$ & $3.16 \pm 0.02 * *$ & $3.07 \pm 0.03 * * *$ \\
\hline Periosteal circumference (mm) & $4.94 \pm 0.03$ & $4.81 \pm 0.03 * *$ & $4.51 \pm 0.03 * * *$ \\
\hline Cortical BMC (mg/mm) & $0.86 \pm 0.01$ & $0.83 \pm 0.01 *$ & $0.64 \pm 0.02 * * *$ \\
\hline Cortical thickness ( $\mu \mathrm{m})$ & $264 \pm 3$ & $262 \pm 2$ & $229 \pm 5 * * *$ \\
\hline
\end{tabular}

Final body and relative liver weight, femoral length, trabecular bone mineral density (Tb. BMD), endocortical and periosteal circumferences, cortical bone mineral content (BMC), and cortical thickness as assessed by pQCT of the femur after 8 days of supplemented and hypervitaminosis A diet. Values represented as mean \pm S.E.M., $n=10$ /group, 1-way ANOVA followed by Dunnett's multiple comparison test vs control. $* P<0.05, * * P<0.01, * * * P<0.001$.

http://joe.endocrinology-journals.org https://doi.org/10.1530/JOE-18-0316
(C) 2018 The authors Published by Bioscientifica Ltd. Printed in Great Britain

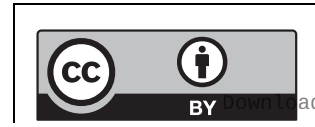

This work is licensed under a Creative Commons Attribution 4.0 Unported License.

ed from Bioscientifica.com at 04/26/2023 10:47:58AM 
A

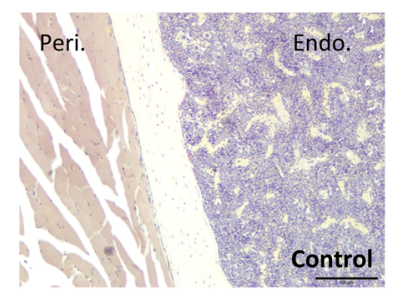

B

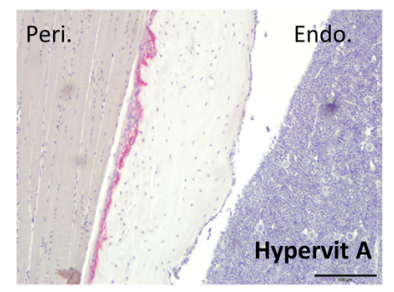

C

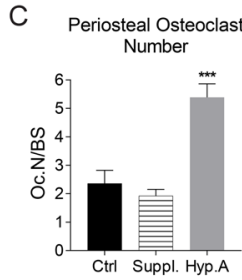

D

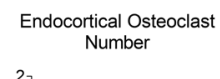

E Trabecular Osteoclast

$\mathrm{F}$
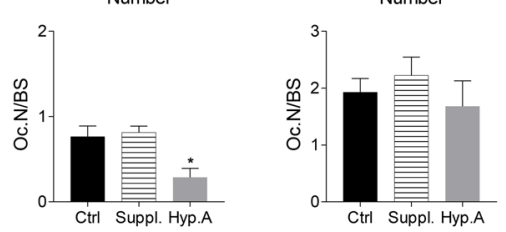

G
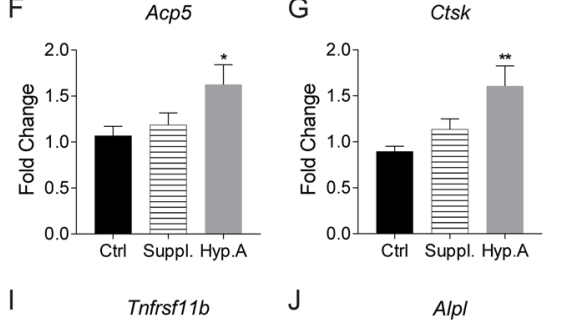

$J$

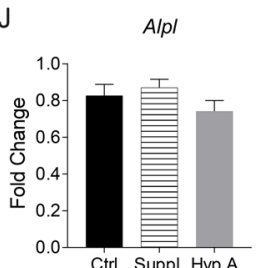

M
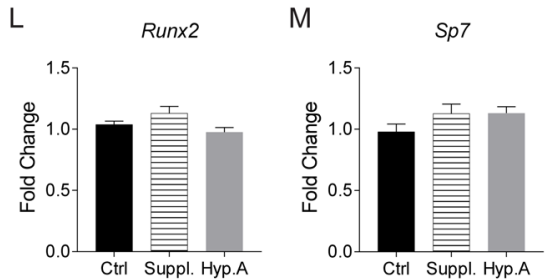

$\mathrm{H}$

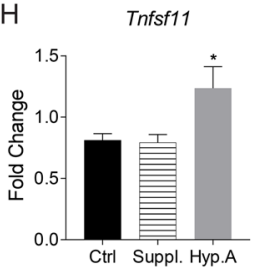

K

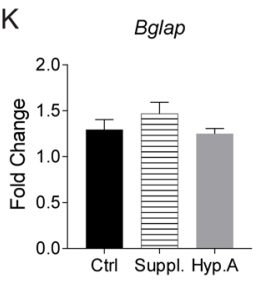

$\mathrm{N}$

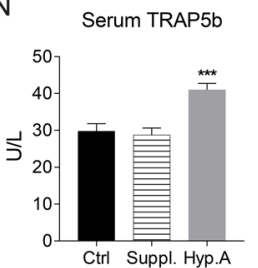

Figure 3

Short duration hypervitaminosis A experiment decreased cortical bone parameters, an effect caused by an increase in osteoclast number. Representative images of TRAP stained sections of femur of $(A)$ control and (B) hypervitaminosis A treated mice for 8 days, scale bars represent $100 \mu \mathrm{m}$. Osteoclast numbers per measured bone surface counted from the (C) periosteum, (D) endosteum, and (E) trabeculae in TRAP stained femur sections of control $(n=10)$, supplemental $(n=9)$, and hypervitaminosis $A(n=5)$ mice after 8 days. Gene expression from cortical bone of the tibia of osteoclastic genes (F) Acp5 (encodes for Trap), (G) Ctsk, (H) Tnfsf11 (encodes for Rankl), (I) Tnfrsf11b (encodes for Opg) and osteoblastic genes (J) Alpl, (K) Bglap (encodes for Osteocalcin), (L) Runx2, and (M) Sp7 (encodes for Osterix) in fold change relative to control. Serum analysis of (N) TRAP5b. Figures displayed as mean \pm S.E.M., 1-way ANOVA followed by Dunnett's multiple comparison test vs control, ${ }^{*} P<0.05, * * P<0.01, * * * P<0.001$, $n=10 /$ group.

UTL dose, when tested separately, did not reach statistical significance.

Supplemented vitamin A diet caused a decrease in periosteal and endocortical circumferences of bone after 8 days, and at 4 and 10 weeks, with the severity of the periosteal phenotype increasing with time. Cortical BMC also mimicked this effect. These findings are consistent with previous studies, regardless of doses, administration or animal model used (Johansson et al. 2002, Kneissel et al. 2005, Lind et al. 2006, 2011, 2013). Cortical thickness significantly decreased after 10 weeks of supplemented vitamin A diet in the present study, and collectively, the results of our study indicate smaller bones.

In human studies, associations have been made between elevated vitamin A intake and decreased bone strength (Melhus et al. 1998, Feskanich et al. 2002, Promislow et al. 2002, Michaelsson et al. 2003); however, it is important to note that not all studies have observed this and on the contrary, inverse (Barker et al. 2005, Maggio et al. 2006) or no (Sowers \& Wallace 1990, Wolf et al. 2005) association between increased vitamin A intake and fracture risk have also been reported. A meta-analysis of 12 prospective studies has recently concluded that both high and low levels of serum retinol, but not intake of $\beta$-carotene, are associated with increased risk of hip fracture, but not the risk of all fractures in the skeleton (Wu et al. 2014). These findings are in agreement with our observations that increased vitamin A decreases cortical but not trabecular bone. Johansson et al. investigated 'subclinical hypervitaminosis A' doses in rats and observed that $180 \mu \mathrm{g}$ retinyl acetate and palmitate/g pellet for 12 weeks resulted in a significant reduction of breaking force compared to controls (Johansson et al. 2002). A short-duration (7-day) hypervitaminosis A (510 $\mu \mathrm{g}$ retinyl acetate and palmitate/g pellet) study in rats done by Lind et al. also observed a reduction in bone strength (Lind et al. 2011). These data, along with our findings, indicate weaker bones with increased vitamin A intake, even at clinically relevant doses although a certain threshold of vitamin A status most likely must be reached. Our supplemented vitamin A dose ( $60 \mu \mathrm{g}$ retinyl acetate/g chow) resulted in reduced bone strength calculated indirectly as polar moment of inertia from pQCT data. Three-point bending also indicated a trend in reduced bone strength (-9\%) after 10 weeks; however, it was not statistically significant.

While a cortical phenotype is evident, both with our clinically relevant doses of vitamin A-treated mice, and previous rat hypervitaminosis A studies, fewer studies have addressed the trabecular bone phenotype.

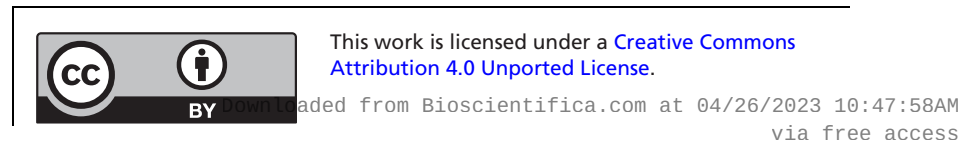


Table 7 Dynamic histology shows vitamin A decreased periosteal bone formation and increased endocortical bone formation after 8 days of supplemented vitamin A diet.

\begin{tabular}{lcc}
\hline & Control $(n=10)$ & Supplemented $(n=8)$ \\
Periosteal & & $18.1 \pm 3.98$ \\
MS/BS $(\%)$ & $23.2 \pm 4.34$ & $0.91 \pm 0.11^{P=0.062}$ \\
MAR $(\mu \mathrm{m} /$ day) & $2.16 \pm 0.55$ & $0.18 \pm 0.06^{*}$ \\
BFR/BS $\left(\mu \mathrm{m}^{3} / \mu \mathrm{m}^{2} /\right.$ day) & $0.47 \pm 0.10$ & $88.3 \pm 1.8$ \\
Endocortical & & $6.50 \pm 0.31^{*}$ \\
MS/BS $(\%)$ & $85.7 \pm 2.4$ & $5.76 \pm 0.36^{*}$ \\
MAR $(\mu \mathrm{m} /$ day) & $5.38 \pm 0.26$ & \\
BFR/BS $\left(\mu \mathrm{m}^{3} / \mu \mathrm{m}^{2} /\right.$ day) & $4.63 \pm 0.30$ & \\
\hline
\end{tabular}

Femur periosteal and endocortical mineralized surface/bone surface (MS/BS), mineral apposition rate (MAR), and bone formation rate/bone surface (BFR/BS) after 8 days of supplemented vitamin A diet obtained from dynamic histomorphometry. Values represented as mean \pm S.E.M. Student's $t$-test. $\star P<0.05$.

Kneissel et al. injected rats with $150 \mu \mathrm{g} / \mathrm{kg}$ of retinoid (Ro 13-6298) once per day for 4 days and found no changes in tibia trabecular BMD based on pQCT (Kneissel et al. 2005). Green et al. gavage-fed mice with ATRA $(5 \mathrm{mg} / \mathrm{kg} /$ day) for 10 days and did not observe changes in trabecular bone volume of the tibia assessed by histomorphometric analysis (Green et al. 2015). On the other hand, Lind et al. observed a statistically significant decrease in femur trabecular BMD when rats were fed excess vitamin A $(510 \mu \mathrm{g}$ retinyl acetate and palmitate/g pellet) for 7 days (Lind et al. 2011), and Wray et al. also observed lower trabecular BMD of the tibia in rats fed adequate ( $4 \mu \mathrm{g}$ retinyl palmitate/g chow) and supplemented $(50 \mu \mathrm{g} / \mathrm{g}$ ) vitamin A diets compared to marginal $(0.35 \mu \mathrm{g} / \mathrm{g})$ vitamin A diet from weaning to 2-3, 8-10 and 18-20 months of age (Wray et al. 2011). Likewise, C57BL/6 female mice fed a diet of $22.5 \mu \mathrm{g}$ retinol/g of chow from weaning to 6 months of age had significantly reduced BV/TV in the vertebra, as demonstrated by Yorgan et al. (2016). This conflicting evidence of excess vitamin A on trabecular bone may partially be explained by administration, dosage, duration, gender and age differences of the rodents used in these investigations, but the effect of vitamin A on trabecular bone remains an area that has been relatively unexplored. We were unable to detect any effect on trabecular bone in the tibia or vertebra of female mice at 4 or 10 weeks using doses of vitamin $A$ that decreased cortical bone mass, and our hypervitaminosis A dose did not significantly alter trabecular bone after 8 days.

A mechanism for the decrease in bone size was not elucidated by gene expression analysis on cortical bone or serum turnover markers. In agreement with these observations, the number of periosteal, endocortical and trabecular osteoclasts in the present study was not significantly affected with supplemented vitamin A diet. Kneissel et al. gave rats subcutaneous injections of $125 \mu \mathrm{g} / \mathrm{kg}$ of retinoid (Ro 13-6298) daily for 4 days. This caused an increase in serum TRAP5b levels due to increased periosteal osteoclast numbers (Kneissel et al. 2005). Lind et al. fed rats $510 \mu \mathrm{g}$ retinyl acetate and palmitate/g pellet for 7 days and also found that a hypervitaminosis A dose increased the number of periosteal osteoclasts. In contrast to Kneissel et al., Lind et al. observed that hypervitaminosis A decreased serum TRAP5b and CTX, possibly due to the reduced number of endocortical osteoclasts found in their study (Lind et al. 2011). Confirming the previous studies in rats (Kneissel et al. 2005, Lind et al. 2011), we found that short-term hypervitaminosis A in mice substantially
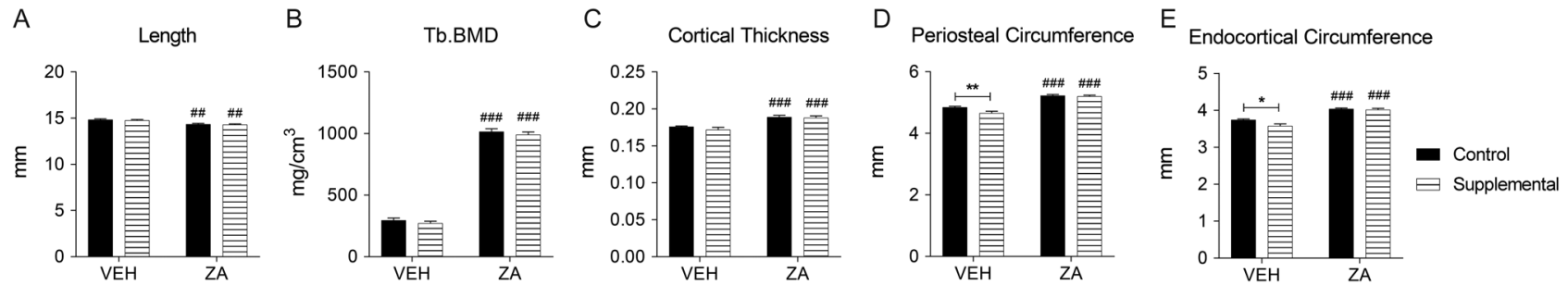

Figure 4

Vitamin A effects on cortical bone were not observed in the presence of bisphosphonate treatment. Femur (A) length, (B) trabecular bone mineral density (Tb. BMD), (C) cortical bone thickness, and (D) periosteal and (E) endocortical circumference as measured by pQCT. Similar results observed in tibia. Values given as mean \pm s.E.M., $n=10 /$ group. Student's $t$-test; Vehicle $(V E H)$ : control vs supplemented, and Zoledronic acid (ZA): control vs supplemented $* P<0.05$, ${ }^{*} P<0.01$. Student's $t$-test ZA treatment vs respective VEH control, $\# P<0.05, \# \# P<0.01, \# \# P<0.001$. 2 -way ANOVA for interaction, $P>0.05$.

http://joe.endocrinology-journals.org https://doi.org/10.1530/JOE-18-0316 (c) 2018 The authors Published by Bioscientifica Ltd. Printed in Great Britain

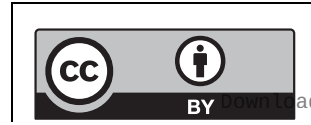

This work is licensed under a Creative Commons Attribution 4.0 Unported License. 
increased osteoclast numbers on the periosteal surface of the long bones and increased serum Trap5b. The enhanced number of osteoclasts was associated with an increased expression of the genes encoding TRAP and cathepsin $\mathrm{K}$ due to the increased expression of the RANKL gene. This is in agreement with our previous ex vivo observations in vitamin A-treated mouse calvarial bones (Conaway et al. 2011). Similar to Lind et al., we also observed that short term hypervitaminosis A, for reasons unknown, decreased endocortical osteoclast numbers.

Based on periosteal MS/BS in the femur and tibia of rats, studies have suggested that the number of active osteoblasts is decreased in hypervitaminosis A treatment (Kneissel et al. 2005, Lind et al. 2013). In one of these studies (Lind et al. 2013), a strong decrease of osteoblastic bone formation was seen in rats fed $510 \mu$ g retinyl acetate and palmitate/g pellet for 7 days. This resulted in a decrease in MAR and BFR in the periosteal bone of the femur. In another study (Kneissel et al. 2005), no decrease of MAR, but a decrease of MS was observed in rats given subcutaneous injections of Ro 13-6298. In the present study, we observed that the supplemental dose of vitamin A in mice also decreased periosteal bone formation at 8 days. In contrast to the observations in the periosteum, treatment with vitamin A for 8 days enhanced bone formation at the endocortical surface, with no change in MS/BS, suggesting increased osteoblast activity. Transient loss of this effect may indicate a new steady state in bone remodeling.

Altogether, our observations suggest that reduced periosteal circumference caused by vitamin A may be due to both increased bone resorption and decreased formation and that the reduced endocortical circumference may be due to both enhanced bone formation and reduced resorption. To elucidate the mechanism involved in the cortical bone phenotype in the present study, we used a bisphosphonate, ZA. Treating mice with ZA or a saline vehicle and supplemented or control vitamin A diets indicated that the effect of vitamin $\mathrm{A}$ on the amount of cortical bone seems to be primarily mediated by osteoclasts, because there was no effect on cortical bone parameters in mice fed vitamin A and treated with ZA. Our observations further suggest that the decreased periosteal bone formation and increased endocortical bone formation that were observed may be secondary to an effect of vitamin A on osteoclasts, but we cannot rule out the possibility that direct effects on bone formation are too small in magnitude to result in significant effects on cortical bone mass.

The present study was conducted in female mice, and since gender differences have been reported in age-related bone loss in mice (Glatt et al. 2007), our results may not necessarily be transferable to male mice. For the present study, we used young mice that were growing for the first 5-6 weeks of vitamin A dietary consumption. It is possible that the effects observed are a combination of bone growth and adult bone remodeling. However, we did not observe any vitamin A effects on bone length measurements. Although bone remodeling in growing mice may not be identical to bone remodeling in adult humans, our observations support the epidemiological findings in humans indicating that vitamin $\mathrm{A}$ is a risk factor for osteoporosis and further suggest that the UTL in humans may need to be re-evaluated in relation to the harmful effects on the skeleton.

\section{Conclusion}

Our results suggest that even clinically relevant doses of vitamin A consumed over a long period of time may have a negative impact on bone phenotype, mainly on cortical bone, with trabecular bone remaining unaffected. These data might help to explain why increased vitamin A intake and enhanced serum retinol appear to be associated with an increased risk of non-vertebral fractures, mainly dependent on cortical bone, but not with risk of vertebral fractures, mainly dependent on trabecular bone.

Supplementary data

This is linked to the online version of the paper at https://doi.org/10.1530/ JOE-18-0316.

Declaration of interest

The authors declare that there is no conflict of interest that could be perceived as prejudicing the impartiality of the research reported.

\section{Funding}

This work was funded by the Marie Curie Initial Training Network (Euroclast, FP7-People-2013-ITN: \#607446), the Swedish Research Council, the ALF/LUA research grant from the Sahlgrenska University Hospital in Gothenburg, the IngaBritt and Arne Lundberg Foundation, the Royal 80Year Fund of King Gustav $\vee$ and Åke Wibergs foundation.

Author contribution statement

Author's roles: Study design: U H L, H H C, P H and V L. Study conduct: V L, K L G, A W, S H W, and P H. Data collection: V L, A K, and J T. Data analysis: V L, H J, and P H. Data interpretation: V L, C O, P H, and U H L. Drafting 
manuscript: V L, P H, and U H L. Revising manuscript content: $V \mathrm{~L}, \mathrm{C} \mathrm{O}, \mathrm{H}$ $H C, P H$, and $U \mathrm{H} L$. Approving final version of manuscript: $V L, K L G, A$ W, S H W, A K, J T, H J, C O, H H C, P H, and U H L. U H L, P H and V L take responsibility for the integrity of the data analysis.

\section{Acknowledgements}

The authors would like to thank Charlotta Uggla, Biljana Aleksic and Anette Hansevi for excellent technical assistance.

\section{References}

Bailey RL, Gahche JJ, Lentino CV, Dwyer JT, Engel JS, Thomas PR, Betz JM, Sempos CT \& Picciano MF 2011 Dietary supplement use in the United States, 2003-2006. Journal of Nutrition 141 261-266. (https://doi.org/10.3945/jn.110.133025)

Ballew C, Bowman BA, Russell RM, Sowell AL, Gillespie C \& National Health and Nutrition Examination Survey (NHANES III) 2001 Serum retinyl esters are not associated with biochemical markers of liver dysfunction in adult participants in the third National Health and Nutrition Examination Survey (NHANES III), 1988-1994. American Journal of Clinical Nutrition 73 934-940. (https://doi.org/10.1093/ ajcn/73.5.934)

Barker ME, McCloskey E, Saha S, Gossiel F, Charlesworth D, Powers HJ \& Blumsohn A 2005 Serum retinoids and beta-carotene as predictors of hip and other fractures in elderly women. Journal of Bone and Mineral Research 20 913-920. (https://doi.org/10.1359/JBMR.050112)

Bouxsein ML, Boyd SK, Christiansen BA, Guldberg RE, Jepsen KJ \& Muller R 2010 Guidelines for assessment of bone microstructure in rodents using micro-computed tomography. Journal of Bone and Mineral Research 25 1468-1486. (https://doi.org/10.1002/jbmr.141)

Conaway HH, Pirhayati A, Persson E, Pettersson U, Svensson O, Lindholm C, Henning P, Tuckermann J \& Lerner UH 2011 Retinoids stimulate periosteal bone resorption by enhancing the protein RANKL, a response inhibited by monomeric glucocorticoid receptor. Journal of Biological Chemistry 286 31425-31436. (https://doi. org/10.1074/jbc.M111.247734)

Conaway HH, Henning P \& Lerner UH 2013 Vitamin A metabolism, action, and role in skeletal homeostasis. Endocrine Reviews 34 766-797. (https://doi.org/10.1210/er.2012-1071)

Croquet V, Pilette C, Lespine A, Vuillemin E, Rousselet MC, Oberti F, Saint Andre JP, Periquet B, Francois S, Ifrah N, et al. 2000 Hepatic hyper-vitaminosis A: importance of retinyl ester level determination. European Journal of Gastroenterology and Hepatology 12 361-364. (https://doi.org/10.1097/00042737-200012030-00016)

de Molon RS, Shimamoto H, Bezouglaia O, Pirih FQ, Dry SM, Kostenuik P, Boyce RW, Dwyer D, Aghaloo TL \& Tetradis S 2015 OPG-Fc but not zoledronic acid discontinuation reverses osteonecrosis of the jaws (ONJ) in mice. Journal of Bone and Mineral Research 30 1627-1640. (https://doi.org/10.1002/jbmr.2490)

Dempster DW, Compston JE, Drezner MK, Glorieux FH, Kanis JA, Malluche H, Meunier PJ, Ott SM, Recker RR \& Parfitt AM 2013 Standardized nomenclature, symbols, and units for bone histomorphometry: a 2012 update of the report of the ASBMR Histomorphometry Nomenclature Committee. Journal of Bone and Mineral Research 28 2-17. (https://doi.org/10.1002/jbmr.1805)

Feskanich D, Singh V, Willett WC \& Colditz GA 2002 Vitamin A intake and hip fractures among postmenopausal women. JAMA 287 47-54. (https://doi.org/10.1001/jama.287.1.47)

Glatt V, Canalis E, Stadmeyer L \& Bouxsein ML 2007 Age-related changes in trabecular architecture differ in female and male C57BL/6J mice. Journal of Bone and Mineral Research 22 1197-1207. (https://doi. org/10.1359/jbmr.070507)
Green AC, Poulton IJ, Vrahnas C, Hausler KD, Walkley CR, Wu JY, Martin TJ, Gillespie MT, Chandraratna RA, Quinn JM, et al. 2015 RARgamma is a negative regulator of osteoclastogenesis. Journal of Steroid Biochemistry and Molecular Biology 150 46-53. (https://doi. org/10.1016/j.jsbmb.2015.03.005)

Green AC, Martin TJ \& Purton LE 2016 The role of vitamin A and retinoic acid receptor signaling in post-natal maintenance of bone. Journal of Steroid Biochemistry and Molecular Biology 155 135-146. (https://doi. org/10.1016/j.jsbmb.2015.09.036)

Henning P, Conaway HH \& Lerner UH 2015 Retinoid receptors in bone and their role in bone remodeling. Frontiers in Endocrinology 631. (https://doi.org/10.3389/fendo.2015.00031)

Hough S, Avioli LV, Muir H, Gelderblom D, Jenkins G, Kurasi H, Slatopolsky E, Bergfeld MA \& Teitelbaum SL 1988 Effects of hypervitaminosis A on the bone and mineral metabolism of the rat. Endocrinology 122 2933-2939. (https://doi.org/10.1210/endo-122-62933)

Johansson S, Lind PM, Hakansson H, Oxlund H, Orberg J \& Melhus H 2002 Subclinical hypervitaminosis A causes fragile bones in rats. Bone 31 685-689. (https://doi.org/10.1016/S8756-3282(02)00910-9)

Kam RKT, Deng Y, Chen Y \& Zhao H 2012 Retinoic acid synthesis and functions in early embryonic development. Cell and Bioscience 211. (https://doi.org/10.1186/2045-3701-2-11)

Kneissel M, Studer A, Cortesi R \& Susa M 2005 Retinoid-induced bone thinning is caused by subperiosteal osteoclast activity in adult rodents. Bone 36 202-214. (https://doi.org/10.1016/j. bone.2004.11.006)

Krasinski SD, Russell RM, Otradovec CL, Sadowski JA, Hartz SC, Jacob RA \& McGandy RB 1989 Relationship of vitamin A and vitamin E intake to fasting plasma retinol, retinol-binding protein, retinyl esters, carotene, alpha-tocopherol, and cholesterol among elderly people and young adults: increased plasma retinyl esters among vitamin A-supplement users. American Journal of Clinical Nutrition 49 112-120. (https://doi.org/10.1093/ajcn/49.1.112)

Lind PM, Johansson S, Ronn M \& Melhus H 2006 Subclinical hypervitaminosis A in rat: measurements of bone mineral density (BMD) do not reveal adverse skeletal changes. Chemico-Biological Interactions 159 73-80. (https://doi.org/10.1016/j.cbi.2005.10.104)

Lind T, Lind PM, Jacobson A, Hu L, Sundqvist A, Risteli J, YebraRodriguez A, Rodriguez-Navarro A, Andersson G, Melhus H, et al. 2011 High dietary intake of retinol leads to bone marrow hypoxia and diaphyseal endosteal mineralization in rats. Bone $\mathbf{4 8} 496-506$. (https://doi.org/10.1016/j.bone.2010.10.169)

Lind T, Sundqvist A, Hu L, Pejler G, Andersson G, Jacobson A \& Melhus H 2013 Vitamin A is a negative regulator of osteoblast mineralization. PLoS ONE $\mathbf{8}$ e82388. (https://doi.org/10.1371/journal. pone.0082388)

Maggio D, Polidori MC, Barabani M, Tufi A, Ruggiero C, Cecchetti R, Aisa MC, Stahl W \& Cherubini A 2006 Low levels of carotenoids and retinol in involutional osteoporosis. Bone 38 244-248. (https://doi. org/10.1016/j.bone.2005.08.003)

Melhus H, Michaelsson K, Kindmark A, Bergstrom R, Holmberg L, Mallmin H, Wolk A \& Ljunghall S 1998 Excessive dietary intake of vitamin $\mathrm{A}$ is associated with reduced bone mineral density and increased risk for hip fracture. Annals of Internal Medicine $\mathbf{1 2 9}$ 770-778. (https://doi.org/10.7326/0003-4819-129-10-199811150$00003)$

Michaelsson K, Lithell H, Vessby B \& Melhus H 2003 Serum retinol levels and the risk of fracture. New England Journal of Medicine 348 287-294. (https://doi.org/10.1056/NEJMoa021171)

Obrochta KM, Kane MA \& Napoli JL 2014 Effects of diet and strain on mouse serum and tissue retinoid concentrations. PLOS ONE 9 e99435. (https://doi.org/10.1371/journal.pone.0099435)

O’Byrne SM \& Blaner WS 2013 Retinol and retinyl esters: biochemistry and physiology. Journal of Lipid Research 54 1731-1743. (https://doi. org/10.1194/jlr.R037648)

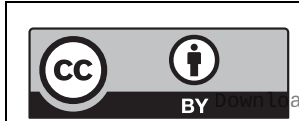

This work is licensed under a Creative Commons Attribution 4.0 Unported License. 
Promislow JH, Goodman-Gruen D, Slymen DJ \& Barrett-Connor E 2002 Retinol intake and bone mineral density in the elderly: the Rancho Bernardo Study. Journal of Bone and Mineral Research 17 1349-1358. (https://doi.org/10.1359/jbmr.2002.17.8.1349)

Sowers MF \& Wallace RB 1990 Retinol, supplemental vitamin A and bone status. Journal of Clinical Epidemiology 43 693-699. (https://doi. org/10.1016/0895-4356(90)90040-V)

Tata JR 2002 Signalling through nuclear receptors. Nature Reviews: Molecular Cell Biology 3 702-710. (https://doi.org/ 10.1038/nrm914)

Trechsel U, Stutzer A \& Fleisch H 1987 Hypercalcemia induced with an arotinoid in thyroparathyroidectomized rats. New model to study bone resorption in vivo. Journal of Clinical Investigation 80 1679-1686. (https://doi.org/10.1172/JCI113257)

Trumbo P, Yates AA, Schlicker S \& Poos M 2001 Dietary reference intakes: vitamin A, vitamin $\mathrm{K}$, arsenic, boron, chromium, copper, iodine, iron, manganese, molybdenum, nickel, silicon, vanadium, and zinc. Journal of The American Dietetic Association 101 294-301. (https://doi. org/10.1016/S0002-8223(01)00078-5)

Whiting SJ \& Lemke B 1999 Excess retinol intake may explain the high incidence of osteoporosis in northern Europe. Nutrition Reviews 57 192-195. (https://doi.org/10.1111/j.1753-4887.1999. tb06942.x)
Wiseman EM, Bar-El Dadon S \& Reifen R 2017 The vicious cycle of vitamin A deficiency: a review. Critical Reviews in Food Science and Nutrition 57 3703-3714. (https://doi.org/10.1080/10408398.2016.11 60362)

Wolf RL, Cauley JA, Pettinger M, Jackson R, Lacroix A, Leboff MS, Lewis CE, Nevitt MC, Simon JA, Stone KL, et al. 2005 Lack of a relation between vitamin and mineral antioxidants and bone mineral density: results from the Women's Health Initiative. American Journal of Clinical Nutrition 82 581-588. (https://doi.org/10.1093/ ajcn.82.3.581)

Wray AE, Okita N \& Ross AC 2011 Cortical and trabecular bone, bone mineral density, and resistance to ex vivo fracture are not altered in response to life-long vitamin A supplementation in aging rats. Journal of Nutrition 141 660-666. (https://doi.org/10.3945/jn.110.132126)

Wu AM, Huang CQ, Lin ZK, Tian NF, Ni WF, Wang XY, Xu HZ \& Chi YL 2014 The relationship between vitamin A and risk of fracture: metaanalysis of prospective studies. Journal of Bone and Mineral Research 29 2032-2039. (https://doi.org/10.1002/jbmr.2237)

Yorgan TA, Heckt T, Rendenbach C, Helmis C, Seitz S, Streichert T, Amling M \& Schinke T 2016 Immediate effects of retinoic acid on gene expression in primary murine osteoblasts. Journal of Bone and Mineral Metabolism 34 161-170. (https://doi.org/10.1007/s00774-0150666-2)

Received in final form 20 September 2018

Accepted 24 September 2018
(C) 2018 The authors Published by Bioscientifica Ltd. Printed in Great Britain
This work is licensed under a Creative Commons Attribution 4.0 Unported License.

ded from Bioscientifica.com at 04/26/2023 10:47:58AM 